\title{
Alterações na qualidade física de um latossolo amarelo sob pastagem
}

\author{
Lorena Chagas TORRES ${ }^{1}$, Katiane Raquel Mendes BARROS², Herdjania Veras de LIMA $^{2 *}$ \\ 1 Escola Superior de Agricultura Luiz de Queiroz- Universidade de São Paulo. Departamento de Solos e Nutrição Mineral de Plantas. Avenida Pádua Dias, 11-Piracicaba- São Paulo, Brasil. \\ 2 Universidade Federal Rural da Amazônia. Instituto de Ciências Agrárias. Avenida Presidente Tancredo Neves, 2501-Belém- Pará, Brasil. \\ * Autor correspondente: herdjania.lima@ufra.edu.br \\ RESUMO
}

Indicadores de qualidade física do solo representam uma importante ferramenta no estudo das alteraçóes que ocorrem no solo em função de açôes de uso e manejo. Objetivou-se com este estudo utilizar o intervalo hídrico ótimo (IHO) e o índice-S (declividade da curva de retenção de água no solo no ponto de inflexão) para quantificar as alteraçóes físicas do solo promovidas pelo sistema de pastagem, tendo como referência um solo sob mata. O estudo foi realizado em um Latossolo Amarelo distroférrico típico sob mata nativa e pastagem, onde foram coletadas amostras deformadas e indeformadas nas profundidades de 0-5 e 25-30 $\mathrm{cm}$, utilizadas para determinação do carbono orgânico do solo e análises físicas. Os valores de índice-S, com exceção do solo sob mata $(0-5 \mathrm{~cm})$, estiveram abaixo do limite sugerido para separar solos com boa e pobre qualidade estrutural. O IHO foi fortemente reduzido no solo sob pastagem, chegando a atingir valor zero. O principal fator limitante para a disponibilidade de água foi a resistência à penetração, que exceto em mata $(0-5 \mathrm{~cm})$, atuou como limite inferior do $\mathrm{IHO}$ em todas as áreas e profundidades avaliadas. Os indicadores de qualidade física do solo, $\mathrm{IHO}$ e índice-S, apresentaram conformidade quanto à avaliação da qualidade física dos solos estudados e indicaram as alteraçóes físicas promovidas no solo sob sistema de pastagem.

PALAVRAS-CHAVE: Manejo do solo, compactação do solo, resistência do solo a penetração

\section{Changes in the physical quality of an Oxisol under grazing system}

\begin{abstract}
Indicators of physical quality of soils are important tools in the study of changes that occur in soils brought about by use and management practices. The objective of this study was to use the least limiting water range (LLWR) and $S$ value (the slope of the soil water retention curve at its inflection point) to quantify soil physical changes promoted by grazing system in comparison with soil under forest vegetation. The study was carried out in an Oxisol under native vegetation and grazing. At these sites disturbed and undisturbed samples were collected at depths of 0-5 and 25-30 cm. The samples were used for chemical and physical analyzes. The $S$-value varied depending on land use. Except under native vegetation (at $0-5 \mathrm{~cm} \mathrm{depth})$, the S-index values were below the limit suggested to permit discriminating between soils with good and poor structural quality. The LLWR was strongly reduced under grazing, reaching zero values. The main limiting factor to water availability was soil resistance, which except under natural vegetation $(0-5 \mathrm{~cm})$, was the factor that determined the lower threshold for water availability in all areas and studied depths. Regarding the assessment of the physical quality of soils, the indicators of soil physical quality, LLWR and S value, yielded similar results and were able to indicated physical changes that occurred in the soil under the grazing system.
\end{abstract}

KEYWORDS: Soil management, soil compaction, soil resistance 


\section{INTRODUÇÃO}

A diminuição da capacidade de infiltração de água, o escoamento superficial, a reduçáo do sistema radicular e a dificuldade de mecanização são considerados indicativos de má qualidade física do solo (Dexter 2004a). Em pastagens, a degradação física do solo pode ocorrer principalmente pela compactação originada pelo pisoteio dos animais, comprometendo mais diretamente as camadas superficiais (Greenwood e Mckenzie 2001). Essas modificações dependem da intensidade do pisoteio, da umidade e do tipo de solo. Os efeitos dos sistemas de pastagens na qualidade física do solo foram reportados mais recentemente na literatura por Leão $e t$ al. (2006), Zhou et al. (2010) e Costa et al. (2012).

O Intervalo Hídrico Ótimo (IHO), proposto Silva et al. (1994) a partir dos conceitos de Letey (1985), tem recebido destaque na avaliação da qualidade física do solo, pois integra em um único parâmetro as variaçôes do conteúdo de água em função da densidade do solo em que ocorrem mínimas limitaçóes ao desenvolvimento de plantas, considerando à porosidade de aeração, resistência à penetração e água disponível. Vários autores atestaram a eficiência do IHO como um indicador da qualidade física dos solos em áreas de pastagens (Leão et al. 2006; Olibone et al. 2010; Calonego et al. 2011).

O índice-S proposto por Dexter (2004a) também é utilizado para avaliar a qualidade física e estrutural do solo, sendo obtido a partir do ponto de inflexão da curva de retenção de água no solo (CRA), que é determinada pela relação entre o conteúdo de água com base em massa e o logaritmo do potencial mátrico do solo. Portanto, o índice-S é definido como valor da inclinação da CRA em seu ponto de inflexão e reflete diretamente a distribuição dos poros de maior frequência no solo.

A utilização do índice-S torna possível a comparação direta de diferentes tipos de solos e sistemas de uso e manejo na avaliação da qualidade física do solo. Dexter (2004b) sugeriu para a avaliação da qualidade física do solo as seguintes categorias de índice-S, baseado em diferentes tipos de solos: $S \geq 0,050$ muito bom, $0,050>S \geq 0,035$ bom, $0,035>S \geq 0,020$ pobre e $0,020>S$ muito pobre. É importante ressaltar que estes valores limitantes de índice-S foram desenvolvidos com base em solos de regióes de clima temperado e, portanto, sua aplicação para solos tropicais deve ser cuidadosa, sendo necessária uma quantidade considerável de estudos que corroborem a eficiência destes valores indicadores ou sugiram novos limites mais adequados para solos desenvolvidos sob clima tropical. Andrade e Stone (2009) verificaram a adequação do índice-S no diagnóstico da qualidade física de solos de Cerrado de diferentes classes texturais e observaram que o valor limite de 0,045 para o índice-S mostrou-se adequado entre solos com boa qualidade estrutural e com grau de degradação, enquanto que solos com $S \leq 0,025$ indicam solos inteiramente degradados fisicamente.

Solos com pobre qualidade física apresentam baixa infiltração de água, escorrimento superficial, baixa aeração, resistência ao crescimento de raízes e dificuldade de mecanização. Enquanto que, boa qualidade física é atribuída a solos com características opostas as citadas acima (Dexter 2004 a). Vários autores confirmaram que o índice-S discrimina os efeitos de uso e manejo sobre a qualidade física do solo, dentre os quais, Tormena et al. (2008), Pereira et al. (2011) e Silva et al. (2011). Neste estudo testou-se a hipótese de que o sistema de pastagem altera a níveis críticos a qualidade física do solo e esta pode ser quantificada pelo IHO e índice-S. Assim, objetivou-se utilizar o IHO e o índice-S para quantificar as alteraçóes físicas do solo promovidas pelo sistema de pastagem, tendo como referência um solo sob mata.

\section{MATERIAL E MÉTODOS}

O estudo foi realizado em duas áreas adjacentes, localizadas no município de Paragominas, Nordeste Paraense. O clima da regiāo é do tipo Aw, segundo a classificação de Köppen, isto é, tropical chuvoso com estação seca bem definida, com temperatura média anual de $26,5^{\circ} \mathrm{C}$. A precipitação pluvial média anual é de $1.800 \mathrm{~mm}$ com duas estaçôes, uma chuvosa que vai de dezembro a maio e outra menos chuvosa de junho a novembro. A umidade relativa do ar varia de $70 \%$ a $90 \%$. O relevo predominante da regiáo varia de plano a suave ondulado, com altitude média em torno de 200 m (Rodrigues et al. 2002).

A amostragem foi realizada em um Latossolo Amarelo distroférrico típico sob mata primária (floresta equatorial subperenifólia) e sob pastagem (Panicum maximum, cultivar Mombaça). O tempo de implantação da pastagem é de oito anos com sistema pastejo extensivo e uma unidade animal por hectare. Anteriormente ao sistema de pastagem, a área foi utilizada para o cultivo de milho.

Em cada área (mata e pastagem) foram coletadas 18 amostras deformadas e 18 indeformadas em superfície $(0-5 \mathrm{~cm})$ e em subsuperfície $(25-30 \mathrm{~cm})$, totalizando 36 amostras indeformadas e 36 amostras deformadas em cada área, divididas nas duas profundidades. O espaçamento entre os pontos de coleta foi de 25 x $30 \mathrm{~m}$, sendo as amostras deformadas e indeformadas coletadas no mesmo ponto. As amostras indeformadas foram coletadas em anéis metálicos de $5 \mathrm{~cm}$ de altura e $5 \mathrm{~cm}$ de diâmetro com auxílio do amostrador de Uhland.

As amostras deformadas foram secas ao ar, destorroadas e peneiradas em malha de $2 \mathrm{~mm}$ para determinação do carbono orgânico do solo e análise granulométrica. A análise granulométrica foi realizada pelo método do densímetro (Gee e Bauder 1986). O teor de carbono orgânico foi determinado por titulação com sulfato ferroso amoniacal a 0,1N Embrapa 
(1997). A distribuição granulométrica, classe textural e carbono orgânico são apresentados na Tabela 1 .

Tabela 1. Distribuição granulométrica, classe textural e carbono orgânico (Corg) do solo sob mata (MT) e pastagem (PA) nas profundidades de 0-5 e $25-30 \mathrm{~cm}$.

\begin{tabular}{lccccc}
\hline \multirow{2}{*}{ Área } & Areia & Silte & Argila & $\mathrm{C}_{\text {org }}$ & \multirow{2}{*}{ Classe textural } \\
\cline { 2 - 5 } & \multicolumn{5}{c}{$\mathrm{g} \mathrm{kg}^{-1}$} \\
MT & 664 & 118 & 219 & 38 & Franco-argilo-arenosa \\
PA & 688 & 125 & 187 & 21 & Franco-arenosa \\
\hline \multicolumn{5}{c}{$25-30 \mathrm{~cm}$} \\
MT & 580 & 140 & 280 & 16 & Franco-argilo-arenosa \\
PA & 619 & 98 & 283 & 6 & Franco-argilo-arenosa \\
\hline
\end{tabular}

As amostras indeformadas foram saturadas em bandejas através da elevação gradual de uma lâmina de água, durante $24 \mathrm{~h}$, e divididas em grupos de seis, sendo três amostras por cada área e profundidade amostrada, em seguida foram submetidas aos potenciais mátricos $(\psi)$ : $-0,03 ;-0,01 \mathrm{MPa}$ em mesa de tensão e $-0,1 ;-0,3 ;-0,7 ;-1,5 \mathrm{MPa}$ em câmaras de pressão (Soil Moisture equipament Co. Santa Barbara, CA, USA), conforme Klute (1986), até atingirem o equilíbrio, cujo momento era definido quando se constatava a completa ausência de drenagem na mesa de tensão e nas câmaras de pressão.

Depois de atingido o equilíbrio das amostras em cada potencial mátrico, foi determinada a massa úmida das amostras. Posteriormente a resistência à penetraçáo foi medida com auxílio de um penetrômetro eletrônico de bancada, montado e desenvolvido no Departamento de Ciência do Solo da ESALQ/USP por Figueiredo et al. (2011). As medidas de resistência foram realizadas no centro de cada amostra, a haste de aço com extremidade cônica (ângulo de $60^{\circ}$ e diâmetro basal de $0,4 \mathrm{~cm}$ ) foi introduzida, a uma velocidade nominal de $10 \mathrm{~mm} \mathrm{~min}{ }^{-1}$, até $4,0 \mathrm{~cm}$ da superfície superior de cada amostra de solo. Foram obtidas um total de 240 mediçôes por amostra de solo, das quais um valor médio foi utilizado.

Em seguida, as amostras de solo foram secas em estufa a $105^{\circ} \mathrm{C}$, durante $24 \mathrm{~h}$, para determinação da massa de solo seco para determinaçáo do conteúdo de água e da densidade do solo conforme Blake e Hartge (1986).

A CRA foi ajustada pelo modelo matemático proposto por van Genuchten (1980) com a restrição $\left(m=1-\frac{1}{n}\right)$, conforme a equação 1 :

$$
\theta=\theta r+\frac{(\theta s-\theta r)}{\left[(1+\alpha \psi)^{n}\right]^{m}}
$$

Onde $\theta$ é a umidade do solo $\left(\mathrm{cm}^{3} \mathrm{~cm}^{-3}\right) ; \theta r$, a umidade residual $\left(\mathrm{cm}^{3} \mathrm{~cm}^{-3}\right) ; \theta$ s, a umidade de saturaçáo $\left(\mathrm{cm}^{3} \mathrm{~cm}^{-3}\right) ; \psi$, o potencial mátrico $(\mathrm{hPa}) ; \alpha, m$, $n$ são os parâmetros empíricos do modelo. A partir dos parâmetros ajustados do modelo de van Genuchten (1980) foi calculado o índice-S conforme Dexter (2004a) (Equação 2):

$$
\text { indice }-S=-n(\theta s-\theta r)\left[1+\frac{1}{m}\right]^{-[1+m]}
$$

Para o cálculo do índice-S as umidades devem ser expressas à base de massa.

A distribuição do diâmetro dos poros foi calculada a partir da derivação da equação 1 (Dexter 2004 a), conforme a equação 3:

$$
\frac{d \theta}{d \ln (\psi)}=-m n\left(\theta_{s}-\theta_{r}\right) \alpha^{n} \psi^{n}\left(1+\alpha \psi^{n}\right)^{-m}
$$

O IHO foi determinado conforme Silva et al. (1994). Os valores críticos para o crescimento das plantas de acordo com o potencial mátrico, resistência do solo e porosidade de aeração foram, respectivamente: a capacidade de campo $\left(\theta_{\mathrm{CC}}\right)$ ou conteúdo de água no potencial mátrico de $-0,01 \mathrm{MPa}$ (Haise et al. 1955), o ponto de murcha permanente $\left(\theta_{\mathrm{PMP}}\right)$ ou conteúdo de água no potencial de -1,5 MPa (Richards e Weaver 1944), o conteúdo de água no solo em que a resistência $\left(\theta_{\mathrm{RP}}\right)$ atinge 2,0 $\mathrm{MPa}$ (Taylor et al. 1966), e o conteúdo de água no solo em que a porosidade de aeração $\left(\theta_{\mathrm{PA}}\right)$ é de 0,10 $\mathrm{cm}^{3} \mathrm{~cm}^{-3}$ (Grable e Siemer 1968).

A relação entre $\theta$ e $\psi$ foi estabelecida a partir do modelo proposto por Silva et al. (1994), considerando sua forma linear, que considera o efeito da densidade do solo (Ds) na retençáo de água, conforme a equação 4 :

$$
\ln \theta=a+b D s+c \ln \psi
$$

Onde Ds é a densidade do solo $\left(\mathrm{Mg} \mathrm{m}^{-3}\right)$ e $a, b$ e $c$ são os parâmetros de ajuste do modelo.

Os dados de resistência à penetração foram ajustados em relação à $\theta$ e à Ds utilizando o modelo proposto por Busscher (1990) em sua forma linear (Equaçáo 5):

$$
\ln R P=\ln d+e \ln \theta+f \ln D s
$$

Onde RP é a resistência à penetração $(\mathrm{MPa})$. Os coeficientes $d$, $e$ e $f$ são os parâmetros do modelo.

Os valores de $\theta_{\mathrm{RP}}$ em que ocorre resistência à penetração de 2,0 MPa foram obtidos por meio do modelo matemático ajustado aos dados de resistência conforme a equaçáo 5 . O valor de $\theta_{\mathrm{PA}}$, em que a porosidade de aeração é de $10 \%$, foi calculado a partir da equaçáo 6 .

$$
\theta P A_{10 \%}=\left[\left(1-\frac{D s}{D p}\right)-0,1\right]
$$


onde $\theta_{\mathrm{PA} 10 \%}$ é a umidade em que a porosidade de aeração é de $10 \%$ ou $0,1 \mathrm{~cm}^{3} \mathrm{~cm}^{-3}$ e Dp é a densidade de partícula $\left(\mathrm{Mg} \mathrm{m}^{-3}\right)$.

O IHO foi calculado como a diferença entre o limite superior e inferior dos conteúdos de água em que ocorrem os parâmetros físicos considerados (Wu et al. 2003). Se o IHO for igual a zero a densidade do solo é conhecida como densidade crítica (Dsc).

\section{RESULTADOS}

As variáveis físicas, Ds, $\theta$ e RP apresentaram variaçôes em seus valores, o que foi importante para a obtenção de bons ajustes das CRA e curvas de resistência do solo (CRS). A RP destacou-se por apresentar elevada variabilidade (Tabela 2).

Os ajustes das curvas de retenção de água no solo (CRA) ao modelo de van Genucthen, para determinação do índice-S, foram realizados utilizando a totalidade dos dados obtidos para cada área e profundidade avaliada $(\mathrm{N}=18)$. Considerando os limites de índice-S propostos por Dexter (2004a, b), observouse na área sob mata $(0-5 \mathrm{~cm})$ valor superior a 0,035 , sugerido como o limite entre solos de boa e pobre qualidade estrutural. As demais áreas estiveram abaixo deste limite, sendo que em pastagem $(0-5$ e $25-30 \mathrm{~cm})$ os valores foram mais próximos de 0,02, valor limite para solos com elevado grau de degradaçáo (Tabela 3). Constatou-se alteraçóes na amplitude das curvas de distribuição do diâmetro dos poros (Figura 1A e B). Em pastagem $(0-5 \mathrm{~cm})$ houve predominância de poros de $48 \mu \mathrm{m}$,

Tabela 2. Estatística descritiva para densidade do solo, umidade do solo e resistência do solo à penetração de raízes em solos sob mata (MT) e pastagem (PA) nas profundidades de 0-5 e 25-30 cm ( $\mathrm{N}=18)$. Ds: densidade do solo; $\theta$ umidade do solo; RP: resistência do solo à penetração; DP: desvio padrão; CV: coeficiente de variação.

\begin{tabular}{|c|c|c|c|c|c|}
\hline Variável & Média & Mínimo & Máximo & $\mathrm{DP}$ & $\mathrm{CV}(\%)$ \\
\hline \multicolumn{6}{|c|}{ MT $(0-5 \mathrm{~cm})$} \\
\hline Ds $\left(\mathrm{Mg} \mathrm{m}^{-3}\right)$ & 1,26 & 1,03 & 1,40 & 0,08 & 6,56 \\
\hline$\theta\left(\mathrm{cm}^{3} \mathrm{~cm}^{-3}\right)$ & 0,23 & 0,11 & 0,40 & 0,09 & 39,46 \\
\hline $\mathrm{RP}(\mathrm{MPa})$ & 1,54 & 0,78 & 2,44 & 0,55 & 34,82 \\
\hline \multicolumn{6}{|c|}{$\mathrm{PA}(0-5 \mathrm{~cm})$} \\
\hline Ds $\left(\mathrm{Mg} \mathrm{m}^{-3}\right)$ & 1,60 & 1,49 & 1,69 & 0,05 & 3,21 \\
\hline$\theta\left(\mathrm{cm}^{3} \mathrm{~cm}^{-3}\right)$ & 0,23 & 0,16 & 0,33 & 0,05 & 22,35 \\
\hline $\mathrm{RP}(\mathrm{MPa})$ & 3,60 & 0,73 & 10,41 & 0,58 & 67,97 \\
\hline \multicolumn{6}{|c|}{ MT $(25-30 \mathrm{~cm})$} \\
\hline Ds $\left(\mathrm{Mg} \mathrm{m}^{-3}\right)$ & 1,45 & 1,28 & 1,57 & 0,08 & 5,62 \\
\hline$\theta\left(\mathrm{cm}^{3} \mathrm{~cm}^{-3}\right)$ & 0,22 & 0,12 & 0,34 & 0,07 & 32,12 \\
\hline $\mathrm{RP}(\mathrm{MPa})$ & 2,37 & 0,80 & 5,72 & 1,46 & 61,82 \\
\hline \multicolumn{6}{|c|}{$\mathrm{PA}(25-30 \mathrm{~cm})$} \\
\hline Ds $\left(\mathrm{Mg} \mathrm{m}^{-3}\right)$ & 1,59 & 1,43 & 1,70 & 0,08 & 5,40 \\
\hline$\theta\left(\mathrm{cm}^{3} \mathrm{~cm}^{-3}\right)$ & 0,23 & 0,15 & 0,33 & 0,06 & 25,25 \\
\hline $\mathrm{RP}(\mathrm{MPa})$ & 3,64 & 0,65 & 10,90 & 3,52 & 96,79 \\
\hline
\end{tabular}

enquanto em mata $(0-5 \mathrm{~cm})$ houve maior quantidade de poros de $180 \mu \mathrm{m}$ (Figura 1A). A $25-30 \mathrm{~cm}$ de profundidade, os poros predominantes em pastagem foram $72 \mu \mathrm{m}$ e em mata de $144 \mu \mathrm{m}$ (Figura 1B).

$\mathrm{Na}$ determinação do IHO, a umidade do solo apresentou correlaçáo positiva com a Ds e negativa com o $\psi$, enquanto que a RP variou positivamente com a Ds e negativamente com o conteúdo de água, em todas as áreas estudadas (Tabela 4).

No solo sob mata $(0-5 \mathrm{~cm})$, o IHO do teve como limite superior e inferior os valores de umidade na capacidade de campo e no ponto de murcha permanente, respectivamente, em toda a variaçáo de Ds, não apresentando, portanto, restriçóes quanto a PA e RP (Figura 2A). Já em pastagem (0-5 $\mathrm{cm}$ ), o limite inferior do IHO foi atribuído à $\theta_{\mathrm{RP}}$, em toda a variação de Ds. A $\theta_{\mathrm{PA}}$ substituiu a $\theta_{\mathrm{CC}}$ no limite superior em

Tabela 3. Parâmetros do ajuste da curva de retenção de água no solo à equação de van Genhuchten e índice-S do solo sob mata (MT) e pastagem (PA) a 0-5 e $25-30 \mathrm{~cm}$ de profundidade. $\theta_{\text {sat }}$ : umidade de saturação; $\theta_{\text {res }}$ : umidade residual; $\alpha, n$ : parâmetros do modelo.

\begin{tabular}{lccccc}
\hline Áreas & \begin{tabular}{c}
$\theta_{\text {sat }}$ \\
\cline { 2 - 4 } $\mathrm{cm}^{3} \mathrm{~cm}^{-3}$
\end{tabular} $\begin{array}{c}\alpha \\
\mathrm{hPa}^{-1}\end{array}$ & $n$ & índice-S \\
\hline \multicolumn{5}{c}{$0-5 \mathrm{~cm}$} \\
MT & 0,387 & 0,108 & 0,012 & 1,446 & 0,048 \\
PA & 0,356 & 0,163 & 0,045 & 1,370 & 0,023 \\
\hline \multicolumn{6}{c}{$25-30 \mathrm{~cm}$} \\
MT & 0,343 & 0,129 & 0,015 & 1,433 & 0,032 \\
PA & 0,352 & 0,162 & 0,029 & 1,444 & 0,026 \\
\hline
\end{tabular}

(*): 0 índice-S foi calculado com base na $\theta$ em $\mathrm{kg} \mathrm{kg}^{-1}$

Tabela 4. Modelos de regressão para as curvas de retenção de água e resistência do solo, com transformações logarítmicas dos solos sob mata (MT) e pastagem (PA) a 0-5 e 25-30 cm de profundidade e respectivos coeficientes de determinação. $\theta$ : umidade do solo $\left(\mathrm{cm}^{3} \mathrm{~cm}^{-3}\right)$; Ds: densidade do solo $(\mathrm{Mg}$ $\left.\mathrm{m}^{-3}\right) ;$ : potencial mátrico (MPa); RP: resistência do solo à penetração(MPa).

\begin{tabular}{|c|c|c|}
\hline Áreas & Modelos de regressão & $r^{2}$ \\
\hline \multicolumn{3}{|c|}{ Curva de Retenção de Água (CRA) } \\
\hline \multicolumn{3}{|c|}{$0-5 \mathrm{~cm}$} \\
\hline MT & $\ln \theta=-2,05617+0,07845 D s-0,1761 \ln \psi$ & 0,88 \\
\hline PA & $\ln \theta=-3,48879+1,08317 D s-0,10049 \ln \psi$ & 0,91 \\
\hline \multicolumn{3}{|c|}{$25-30 \mathrm{~cm}$} \\
\hline MT & $\ln \theta=-2,78224+0,60178 D s-0,13592 \ln \psi$ & 0,82 \\
\hline PA & $\ln \theta=-2,13426+0,22574 \mathrm{Ds}-0,10294 \ln \psi$ & 0,83 \\
\hline \multicolumn{3}{|c|}{ Curva de Resistência do Solo (CRS) } \\
\hline \multicolumn{3}{|c|}{$0-5 \mathrm{~cm}$} \\
\hline MT & $\operatorname{InRP}=0,43146-0,77384 \operatorname{In} \theta+0,26554 \operatorname{InDs}$ & 0,74 \\
\hline PA & $\operatorname{InRP}=0,00303-2,30273 \ln \theta+7,26962 \operatorname{lnDs}$ & 0,71 \\
\hline \multicolumn{3}{|c|}{$25-30 \mathrm{~cm}$} \\
\hline MT & $\operatorname{lnRP}=0,03871-1,5022 \ln \theta+4,3144 \operatorname{lnDs}$ & 0,86 \\
\hline PA & $\operatorname{InRP}=0,00343-2,71689 \ln \theta+5,28205 \operatorname{lnDs}$ & 0,72 \\
\hline
\end{tabular}




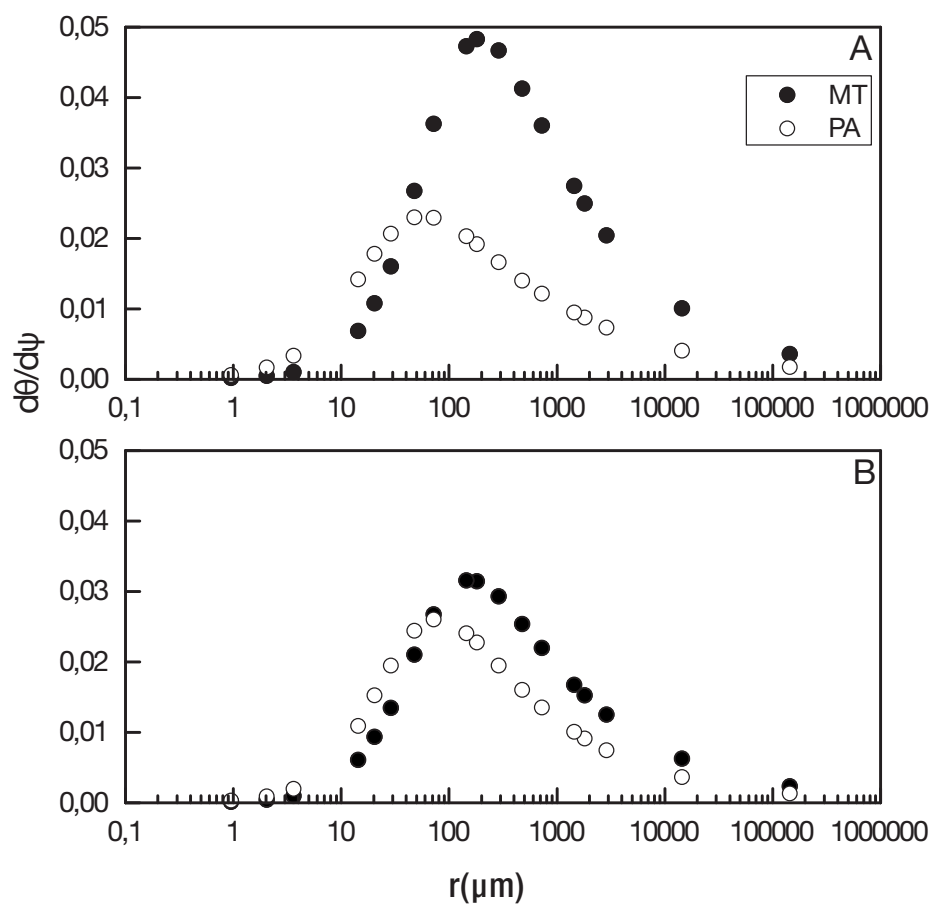

Figura 1. Distribuição do raio dos poros do solo (r) nas áreas sob mata (MT) e pastagem (PA) a 0-5 cm (A) e 25-30 cm de profundidade (B).
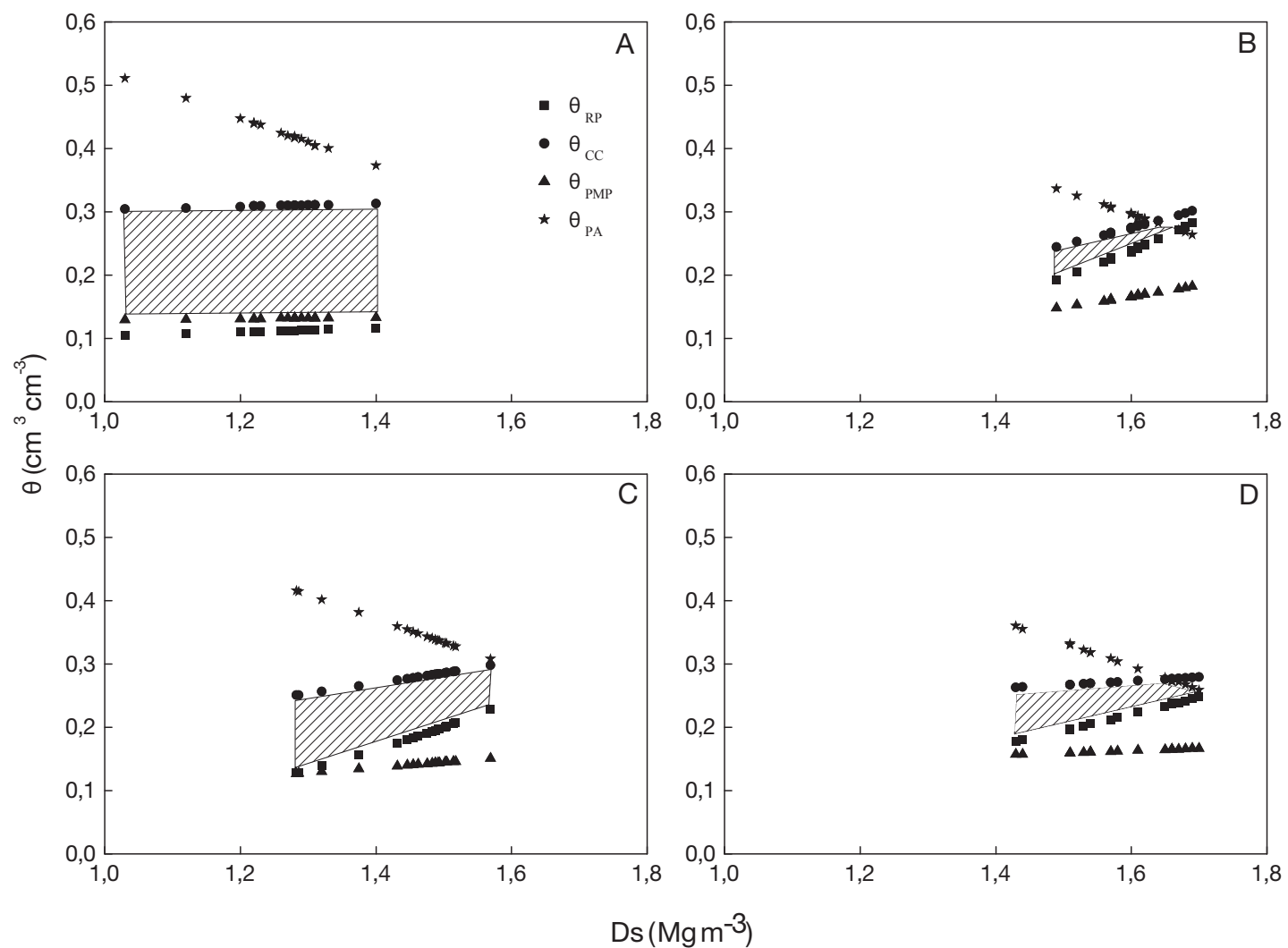

Figura 2. Variação do conteúdo de água ( $\theta$ ) em função da densidade do solo (Ds) em área sob mata (MT) e pastagem (PA) a 0-5 cm (A e B) e 25-30 cm de profundidade (C e D), respectivamente. A área hachurada representa o Intervalo hídrico ótimo ( $\mathrm{HO})$. 
Ds de $1,62 \mathrm{Mg} \mathrm{m}^{-3}$ e a partir da Ds de $1,67 \mathrm{Mg} \mathrm{m}^{-3}$ o IHO foi nulo (Figura 2B).

$\mathrm{Na}$ profundidade de $25-30 \mathrm{~cm}$, as áreas amostradas apresentaram limitaçóes quanto à $\theta_{\mathrm{RP}}$ em toda a variação de Ds. A $\theta_{\mathrm{PA}} \leq 10 \%$ não foi restritiva na área sob mata, enquanto que, sob pastagem a $\theta_{\mathrm{PA}}$ substituiu a $\theta_{\mathrm{CC}}$ como limite superior a partir da Ds de $1,66 \mathrm{Mg} \mathrm{m}^{-3}$ (Figura $2 \mathrm{C}, \mathrm{D}$ ).

De modo geral, a RP apresentou influência marcante nas restriçóes ao crescimento de plantas. Exceto em mata $(0-5 \mathrm{~cm})$, o limite inferior de água disponível referente ao ponto de murcha permanente apresentou pouca influência na determinação da disponibilidade de água no solo, pois, foi substituído pelo conteúdo de água em que a RP restringe o crescimento de plantas, mesmo em baixos valores de Ds (Figura 2B, C, D). Em mata $(0-5$ e $25-30 \mathrm{~cm})$ não foram observados valores de Dsc, valor atingido quando o IHO for zero (Figura 2 A, C). No entanto, em pastagem (0-5 e 25-30 $\mathrm{cm}$ ) alguns valores de Ds ultrapassaram a Dsc (Figura 2 B, D).

\section{DISCUSSÃO}

As variaçôes nos valores de Ds estáo associadas, provavelmente, à heterogeneidade entre os pontos de amostragem distribuídos em campo, enquanto que, para os valores de RP as variaçóes se devem ao gradiente de umidade imposto e as variaçóes nos valores de densidade do solo. Blainsky et al. (2012) obtiveram elevados CV ao estudar essa relação, enfatizando a importância da padronização do conteúdo de água na determinação e interpretação da RP.

$\mathrm{O}$ alto valor de índice-S no solo sob mata na profundidade de $0-5 \mathrm{~cm}$ (Tabela 3 ) indicou uma grande quantidade de poros que drenam a água no ponto de inflexáo, ou seja, aqueles que estão no pico da curva de distribuiçáo do diâmetro dos poros (Dexter, 2004a).

No solo sob pastagem observou-se o menor valor de índice-S, estando associado à maior quantidade de poros de menor diâmetro, que reduz a inclinação da CRA (Dexter 2004a). Tais resultados podem indicar a influência do pisoteio de animais e do tráfego de máquinas, em condiçōes de umidade inadequada, na porosidade responsável pelo movimento rápido da água no solo, com consequente aumento da retenção de água no solo sob pastagem.

Considerando a profundidade de $25-30 \mathrm{~cm}$, no solo sob mata o valor de índice-S, mesmo abaixo do limite de 0,035 , foi superior aquele observado no solo sob pastagem. Os valores de índice-S abaixo do limite de 0,035 , mesmo no solo sob mata, indicam uma característica natural da área de estudo, que pode ter sido acentuada pelo uso do solo.

Pereira et al. (2012), ao estudarem solos da região nordeste, obtiveram valores de índice-S de 0,048 e 0,037 para solos sob condiçóes naturais e 0,021 e 0,016 em pastagem na camada
$5-10 \mathrm{~cm} \mathrm{e} 20-25 \mathrm{~cm}$, respectivamente, e ressaltam as alteraçôes nas características físico-hidrológicas do solo causada pelo intenso trafego de máquinas e animais.

As diferenças observadas entre os valores de índice-S podem ter influência também do carbono orgânico do solo $\left(\mathrm{C}_{\text {org }}\right)$, já que em mata os teores de $\mathrm{C}_{\text {org }}$ foram superiores aos teores em pastagem, o que pode ter contribuído para um melhor arranjamento do espaço poroso e, consequentemente, melhores condiçóes físicas para o crescimento de plantas. Dexter (2004a) e Streck et al. (2008) observaram forte relação entre os valores de índice-S e matéria orgânica do solo.

As alteraçóes observadas na amplitude das curvas de distribuição do diâmetro dos poros (Figura 1A, B) confirmaram as modificaçóes no raio dos poros predominantes, com aumento de poros de menor tamanho em funçáo dos efeitos de uso e manejo do solo, sendo estas alteraçóes, mais evidentes na camada superficial. A partir distribuiçáo do tamanho de poros proposta por Koorevaar et al. (1983), que estabelece como macroporos $(>50 \mathrm{~mm})$, mesoporos $(15 \leq \mathrm{r} \leq 50 \mathrm{~mm})$ e microporos $(<15 \mathrm{~mm})$, observou-se que no solo sob pastagem $(0-5 \mathrm{~cm})$ houve predominância de poros da classe dos mesoporos, enquanto que sob mata $(0-5$ e $25-30 \mathrm{~cm})$ e pastagem $(25-30 \mathrm{~cm})$ observou-se maiores quantidades de poros incluídos na classe dos macroporos.

Na determinação do Intervalo Hídrico Ótimo, os modelos matemáticos ajustados em suas formas linearizadas explicaram acima de $80 \%$ e $70 \%$, respectivamente, a variação do conteúdo de água (CRA) e a curva de resistência do solo (CRS) nas áreas e profundidades avaliadas. A correlaçáo positiva de $\theta$ com a Ds e negativa com o $\psi$ (Tabela 4), pode ser explicada pelo aumento da Ds, que altera a distribuiçáa do diâmetro dos poros, ocasionando aumento no volume de poros com menor diâmetro e consequentemente aumento da retenção de água, estando em concordância com os resultados de Araújo $e t$ al. (2004), Leão et al. (2004) e Leấo et al. (2006). O aumento da $\mathrm{RP}$, devido ao aumento da Ds e decréscimo da $\theta$ (Tabela 4), está relacionado com a reduçáo do efeito lubrificante da água e, consequentemente, acréscimo das forças de coesão entre as partículas do solo (Petean et al. 2010). Esses resultados estáo de acordo com Calonego et al. (2011) e Medeiros et al. (2011).

$\mathrm{O}$ IHO do solo sob mata $(0-5 \mathrm{~cm})$ sendo limitado pela $\theta_{\mathrm{CC}}$ e $\theta_{\text {PMP }}$ em toda a faixa de Ds (Figura 2A) indicou que a aeração manteve-se em níveis satisfatórios quando o solo atingiu a CC, considerando o valor mínimo de $10 \%$ de aeraçáo, e que a RP não alcançou valores impeditivos ao crescimento das plantas. Em pastagem $(0-5 \mathrm{~cm})$ a forte restrição causada pela $\theta_{\mathrm{RP}}$ e $\theta_{\mathrm{PA}}$ ao $\mathrm{IHO}$, que chegou a atingir valor zero, indicou sérias limitaçóes ao crescimento radicular, principalmente, em baixos conteúdos de água (Figura 2B). Tais resultados indicam o efeito da compactaçáo do solo em pastagem, já que, com o aumento da Ds, a porosidade de aeração se torna 
restrita e a resistência do solo aumenta. No solo sob mata $(25-30 \mathrm{~cm})$ a restrição causada pela $\theta_{\mathrm{Rp}}$ associada às limitaçôes observadas a partir do índice-S podem indicar que esta seja uma característica natural da camada subsuperficial (25-30 $\mathrm{cm}$ ) desta área de estudo, que foi intensificada pelo sistema de uso e manejo, conforme observado no solo sob pastagem $(25-30 \mathrm{~cm})$, onde o IHO foi restringido tanto pela $\theta_{\mathrm{RP}}$ quanto pela $\theta_{\mathrm{PA}}$, e o valor de índice-S foi de 0,026 , indicando baixa qualidade estrutural.

A forte influência da RP como fator limitante ao crescimento de plantas tem sido encontrada em muitos estudos com IHO em solos cultivados com diferentes texturas e condiçóes de manejo, em que os valores de $\theta$ tem que ser mantidos em níveis altos para que a RP não atinja valores limitantes, dentre os quais destacam-se, Cavalieri et al. (2011) e Lima et al. (2012). Pereira et al. (2012) ressaltam que a grande influência de fatores como a resistência do solo e a porosidade de aeração na disponibilidade de água confirmam que o conceito de água disponível não é suficiente para estimar os adequados conteúdos de água no solo para o melhor desenvolvimento das plantas. A tendência de redução do IHO com o aumento da Ds mais evidente no solo cultivado, onde o IHO atingiu valor zero, indicou o efeito prejudicial, causado por açôes de manejo, na qualidade física do solo. Em pastagem $(0-5 \mathrm{~cm}$ e $25-30 \mathrm{~cm})$ a ocorrência de valores de Ds $>$ Dsc (Figura $3 \mathrm{~A}, \mathrm{~B}$ ), confirmam a pobre qualidade física do solo nesta condição. Solos sob cultivo de pastagens normalmente apresentam valores de Ds>Dsc, conforme constatado por Leão et al. (2006) e Pereira et al. (2012).

\section{CONCLUSÕES}

Os indicadores de qualidade física do solo, IHO e índice-S, apresentam conformidade quanto à avaliação da qualidade física dos solos estudados e indicam as alteraçóes físicas promovidas no solo sob sistema de pastagem.

\section{AGRADECIMENTOS}

Ao Programa de Pós-graduação em Agronomia da Universidade Federal Rural da Amazônia -UFRA e ao Conselho Nacional de Desenvolvimento Científico e Tecnológico - CNPq, pela concessão da bolsa de estudo. Ao Departamento de Ciência do Solo da "Escola de Agricultura Luiz de Queiroz” - ESALQ/USP, pelo apoio na realização deste trabalho.

\section{BIBLIOGRAFIA CITADA}

Andrade, R. S.; Stone, L. F. 2009. Índice S como indicador da qualidade física de solos do cerrado. Revista Brasileira de Engenharia Agrícola e Ambiental, 13:382-388.
Araújo, M. A.; Tormena, C. A.; Silva, A. P. 2004. Propriedades físicas de um Latossolo Vermelho Distrófico cultivado e sob mata nativa. Revista Brasileira de Ciência do Solo, 28:337-345.

Blainski, E; Tormena, C. T.; Guimarães, R. M. L.; Nanni, M. R. 2012. Qualidade Física de um Latossolo Sob Plantio Direto Influenciada pela Cobertura do Solo. Revista Brasileira de Ciência do Solo, 36:79-87.

Blake, G. R.; Hartge, K. H. 1986. Bulk density. In: Klute, A. (Ed.). Methods of soil analysis. Part 1: Physical and mineralogical methods. 2 ed. American Society of Agronomy - Madison, Wisconsin p.363-375

Busscher, W.J. 1990. Adjustment of flat-tipped penetrometer resistance data to a common water content. American Society of Agricultural Engineers, 33:519-524.

Calonego, J.C.; Borghi, E.; Crusciol, C. A. C. 2011. Intervalo hídrico ótimo e compactação do solo com cultivo consorciado de milho e braquiária. Revista Brasileira de Ciência do Solo, 35:2183-2190.

Cavalieri, K. M. V.; Carvalho, L. A.; Silva, A. P. S.; Libardi, P. L.; Tormena, C. A. 2011. Qualidade física de três solos sob colheita mecanizada de cana-de-açúcar. Revista Brasileira de Ciência do Solo, 35:1541-1549.

Costa, M. A. T.; Tormena, C. A.; Lugão, S. M. B.; Fidalski, J.; Nascimento, W. G.; Medeiros, F. M. 2012. Resistência do solo à penetração e produção de raízes e de forragem em diferentes níveis de intensificação do pastejo. Revista Brasileira de Ciência do Solo, 36:993-1004.

Dexter, A.R. 2004a. Soil physical quality Part I. Theory, effects of soil texture, density, and organic matter, and effects on root growth. Geoderma, 120:201-214.

Dexter, A.R. 2004b. Soil physical quality: Part III. Unsaturated hydraulic conductivity and general conclusions about S-theory. Geoderma, 120:227-239.

Empresa Brasileira de Pesquisa Agropecuária. 1997. Manual de métodos e análise de solo. 2. ed. Rio de Janeiro, 1997, 212p.

Figueiredo, G. C.; Silva, A.P.; Tormena, C. A.; Giarola, N. F. B.; Moraes, S. O.; Almeida, B. G. 2011. Desenvolvimento de um consolidômetro pneumático: modelagem da compactação, penetrometria e resistência tênsil de agregados de solo. Revista Brasileira de Ciência do Solo, 35:389-402.

Gee, G.W.; Bauder, J.W. 1986. Particle-size analysis. In: KLUTE, A. (Ed.). Methods of soil analysis: Physical and mineralogical methods. 2 da ed. Madison, American Society of Agronomy, Part 1. p.383-411.

Grable, A.R.; Siemer, E.G. 1968. Effects of bulk density, aggregate size, and soil water suction on oxygen diffusion, redox potential and elongation of corns roots. Soil Science Society of America Proceedings, 32:180-186.

Greenwood, K. L.; Mckenzie, B. M. 2001. Grazing effects on soil physical properties and the consequences for pastures: a review. Australian Journal of Experimental Agriculture, 41:1231- 1250.

Haise, H.R.; Haas, H.J.; Jensen, L.R. 1955. Soil moisture studies of some Great Plain soils: II. Field capacity as related to $1 / 3$-atmosphere percentage and "minimum point" as related 
to 15- and 26-atmosphere percentages. Soil Science Society of America Proceedings, 34:20-25.

Klute, A. 1986. Water retention: laboratory methods. In: KLUTE, A. (Ed.). Methods of soil analysis. Part 1: Physical and mineralogical methods. 2 da ed. Madison, American Society of Agronomy, p.635-660.

Koorevaar, P.; Menelik, G.; Dirksen, C. 1983. Elements of soil physics. Developments in Soil Science, 1983, vol. 13. Elsevier, Amsterdam.

Leão, T.P.; Silva, A.P.; MACEDO, M.C.M.; IMHOFF, S.; EUCLIDES, V.P.B. 2004. Intervalo hídrico ótimo na avaliação de sistemas de pastejo contínuo e rotacionado. Revista Brasileira de Ciência do Solo, 28: 415-423.

Leão, T.P.; Silva, A.P.; Macedo, M.C.M.; Imhoff, S.; Euclides, V.P.B. 2006. Least limiting water range: A potential indicator of changes in near-surface soil physical quality after the conversion of Brazilian Savanna into pasture. Soil \& Tillage Research, 88:279-285.

Letey, J. 1985. Relationship between soil physical properties and crop production. Advances in Soil Science, 1: 277-294.

Lima, V. M. P.; Oliveira, G. C.; Serafim, M. E.; Curi, N.; Evangelista, A. R. 2012. Intervalo Hídrico Ótimo como Indicador de melhoria da Qualidade Estrutural de Latossolo Degradado. Revista Brasileira de Ciência do Solo, 36:71-78.

Medeiros, J. C.; Silva, A. P. S.; Cerri C. E. P.; Giarola, N. F. B.; Figueiredo G. C.; Fracetto, F. J. C. 2011. Linking physical quality and $\mathrm{CO}_{2}$ emissions under long-term no-till and conventional-till in a subtropical soil in Brazil. Plant Soil, 338:5-15.

Olibone, D.; Encide-Olibone, A. P.; Rosolem C. A. 2010. Least limiting water range and crop yields as affected by crop rotations and tillage. Soil Use and Management, 26:485-493.

Pereira, F. S.; Andrioli, I.; Pereira, F. de S.; Oliveira, P. R.; Centurion, J. F.; Falqueto, R. J.; Martins, A.L. da S. 2011. Qualidade física de um latossolo vermelho submetido a sistemas de manejo avaliado pelo índice-S. Revista Brasileira de Ciência do Solo, 35:87-95.

Pereira, V. P.; Ortiz-Escobar, M. E.; Rocha, G. C.; Assis Júnior, R. N.; Oliveira, T. S. 2012. Evaluation of soil physical quality of irrigated agroecosystems in a semi-arid region of North-eastern Brazil. Soil Research, 50: 455-464.
Petean, L. P.; Tormena, C.A.; Alves, S. J. 2010. Intervalo hídrico ótimo de um latossolo vermelho distroférrico sob plantio direto em sistema de integração lavoura-pecuária. Revista Brasileira de Ciência do Solo, 34:1515-1526.

Richards, L.A.; Weaver, L.R. 1944. Fifteen atmosphere percentage as related to the permanent wilting point. Soil Science, 56:331-339.

Rodrigues, T.E.; Valente, M.A.; Gama, J.R.N.F.; Oliveira Júnior, R.C.; Santos, P.L.; Silva, J.L. 2002. Zoneamento Agroecológico do município de Paragominas, Estado do Pará. Boletim-Técnico Belém: Embrapa Amazônia Oriental, p.64.

Silva, A.P.; Kay, B.D.; Perfect, E. 1994. Characterization of the least limiting water range. Soil Science Society of America Journal, 58:1775-1781.

Silva, G.L.; Lima, H.V.; Campanha, M.M.; Gilkes, R.J.; Oliveira, T.S. 2011. Soil physical quality of Luvisols under agroforestry, natural vegetation and conventional crop management systems in the Brazilian semi-arid region. Geoderma 167:61-70.

Streck, C.A.; Reinert, D.J.; Reichert, J.M.; Horn, R. 2008. Relaçóes do parâmetro $S$ para algumas propriedades físicas de solos do Sul do Brasil. Revista Brasileira de Ciência do Solo, 32: 2603-2612.

Taylor, H.M.; Robertson, G.M.; Parker, J.J. 1966. Soil strength root penetration relations for medium to coarse textured soil materials. Soil Science, 102:18-22.

Tormena, C.A.; Silva, A.P.; Imhoff, S.D.C.; Dexter, A.R. 2008. Quantification of the soil physical quality of a tropical Oxisol using the $S$ index. Scientia Agricola, 65:56-60.

van Genuchten, M.TH. 1980. A Closed-Form equation for predicting the hydraulic conductivity of unsaturated soils. Soil Science Society of America Journal, 44:892-897.

Wu, L.; Feng, G.; Letey, J.; Ferguson, L.; Mitchell, J.; McculloughSanden, B.; Markegard, G. 2003. Soil management effects on the non limiting water range. Geoderma, 114:401-414.

Zhou, Z. C; Gan, Z. T.; Shangguan, Z. P.; Dong, Z. B. 2010. Effects of grazing on soil physical properties and soil erodibility in semiarid grassland of the Northern Loess Plateau (China). Catena, 82:87-91.

Recebido em 01/10/2013

Aceito em 01/04/2014 